\title{
Invisible college within triple helix and social network studies in the age of big data
}

\author{
Han Woo Park ${ }^{1}$ \\ Published online: 13 January 2020 \\ (c) Springer Nature B.V. 2020
}

In this special issue, as the title describes, we collected a set of articles that have long influenced the birth, development, and future of the WATEF (World Association for Triple Helix and Future Strategy studies). The WATEF was founded on the basis of shared intellectual foundation in using the triple helix-based innovation model and social network analysis in Asia. The term 'invisible college' (Wagner 2008) has been used often to describe a small community of researchers who interact with each other and exchange knowledge for building common scholarship, even if they do not always have face-to-face contact. The invisible college continues to sustain scholarship in terms of citations and co-authorship.

The evolution of WATEF closely follows the concept of invisible college. It started with the DISC (Data, Innovation, Social Network, and Convergence) conference (formerly the Daegu-Gyeongbuk International Social Network Conference) held in 2014 and has become a scholarly platform for incubating social-cultural-technical network science projects in the big data era. The DISC conference was the first international event in South Korea endorsed by the Triple Helix Association (THA) and the International Network for Social Network Analysis (INSNA) in the east. As ideas and information among DISC participants are increasingly communicated through multiple networked channels, the WATEF started publishing special issues in prestigious journals including Technological Forecasting and Social Change (Xu et al. 2016), Scientometrics (Khan and Park 2012; Chung and Park 2014), and Quality and Quantity (Lee and Park 2015; Chung and Park 2018; Xu and Park 2017).

Following this tradition, this year's special issue has also collected an excellent mix of articles that represent both the global scholarly network and methodological orientation of the WATEF. The lead article by Inga Ivanova, Nataliya Smorodinskaya, and Loet Leydesdorff proposes a modified index to evaluate competitive advantages and growth potentials of national economies using bipartite country-network data. Their research is mostly devoted to developing an integrated index to measure economic complexity in our nonlinear post-industrial society.

Further investigating how indicators can relate to measurement, Manuela Hartwig and Leslie Tkach-Kawasaki examine the effects of measured results. Using an integrated method involving an innovative survey instrument and actor-network approach, they

Han Woo Park

hanpark@ynu.ac.kr

1 Department of Media and Information, Interdisciplinary Graduate Program of Digital Convergence Business, Cyber Emotions Research Center, YeungNam University, Gyeong-San, Korea 
were able to reveal hidden structures in policymaking processes in Germany regarding energy policy decisions during Japan's pre- and post-Fukushima period.

Next, we have included papers investigating semantic network analysis from the perspectives of various fields. The growing importance of social network analysis has led to the emergence of a rich body of network-based text analytics. More recently, machine learning techniques have appeared as new methods for semantic analysis in the age of big data. The beauty of semantic network analysis lies in deconstructing hidden meanings embedded in textual messages exchanged via social media and the dynamic characteristics of the codified knowledge inherent in those texts.

Related to this trend, James Danowski and Kenneth Riopelle have expanded the breadth of a traditional semantic network approach, reflecting today's rapid advancements in machine learning. Instead of using a stop-word list, for example, they used a start-word list to construct scales to measure constructs and created semantic indices with high predictive validity based on a natural-language corpus containing 56 million words. Similarly, Nicholas Evangelopoulos and Yasaman Amirkiaee use Latent Semantic Analysis (LSA), a more traditional than Latent Dirichlet Allocation (LDA), for their analysis of published articles in information systems and operations management fields.

Breaking new ground in this area, Joseph Woelfel contends that even though the (spatial) techniques we have performed so far have non-Euclidean properties, the Euclidean space is misused. As a result, he suggests that the cognitive and cultural processes resulting from network analysis, pattern recognition, and machine learning, commit mistakes in transforming coordinate systems in non-Euclidean configurations, seriously distorting almost all measurement values in Euclidean space.

According to Piete Stek, the area of visual analytics has been rapidly growing in popularity in research and practice, and can be applied in diverse academic applications. One of the most powerful techniques of data visualization is the heat map methodology based on the Kernel Density Estimation (KDE) method. The KDE, a spatial interpolation technique, offers certain advantages over the cluster method in terms of a laborintensive process when assigning patent addresses to particular cities and then combining neighboring cities into the same cluster.

Sejung Park and Han Woo Park also mapped the interconnection between top-level domains, including country codes and 53 cryptocurrency websites, using a Quantum Geographic Information Systems (QGIS) application. During this process, they found that web traffic and online network metrics were valuable indicators of the financial performance of cryptocurrencies including market capitalization and trading volume.

In our volume's final paper, Miyoung Chong and HaeJung Kim examined a Twitter network through a combined approach of quantitative network analysis and qualitative investigation. Using the \#prayforparis, the authors were able to identify social-media influentials with high centrality measures and uncovered their social roles based on structural signatures of the Twitter network. The findings were discussed drawing on the diffusion of innovation theory.

In summary, the current issue contains eight research articles that have been rigorously reviewed for inclusion in this special section. To ensure objectivity and fairness, one paper co-authored by the guest editor for this issue has been handled separately by the former Editor-in-Chief of the journal. This issue provides comprehensive coverage of important methods involving the concept of triple helix and social networks using large-scale and unstructured data. 


\section{References}

Chung, C.J., Park, H.W.: Mapping triple helix innovation in developing and transitional economies: webometrics, scientometrics, and informetrics. Scientometrics 99(1), 1-4 (2014)

Chung, C.J., Park, H.W.: Beyond data, innovation, social network, and convergence. Qual. Quant. 52(2), 515-518 (2018)

Khan, G.F., Park, H.W.: Editorial: triple-helix and innovation in Asia using scientometrics, webometrics, informetrics. Scientometrics. 90(1), 1-7 (2012)

Lee, Y.-O., Park, H.W.: Triple helix approaches and social network perspectives. Qual. Quant. 49(4), 13231324 (2015)

Wagner, Caroline S.: The New Invisible College: Science for Development. Brooking Press, Washington DC. (2008)

Xu, W.W., Park, H.W.: Versatile networks in combining qualitative and quantitative data. Qual. Quant. 51(3), 959-961 (2017)

Xu, W.W., Cho, I. H., Park, H.W.: Computational social studies in innovations, collaborations and future strategies: Investigating socio-technical interactions using biggish data. Technol. Forecast. Soc. Chang. 100(110), 1-2 (2016)

Publisher's Note Springer Nature remains neutral with regard to jurisdictional claims in published maps and institutional affiliations. 